\title{
Delineating inflammatory bowel disease through transcriptomic studies: current review of progress and evidence
}

\author{
Seow-Neng Chan ${ }^{1}$, Eden Ngah Den Low ${ }^{1}$, Raja Affendi Raja Ali ${ }^{2}$, Norfilza Mohd Mokhtar ${ }^{1}$ \\ ${ }^{1}$ Department of Physiology and ${ }^{2}$ Gastroenterology Unit, Department of Medicine, Faculty of Medicine, Universiti Kebangsaan Malaysia Medical \\ Centre, Kuala Lumpur, Malaysia
}

Inflammatory bowel disease (IBD), which comprises of Crohn's disease and ulcerative colitis, is an idiopathic relapsing and remitting disease in which the interplay of different environment, microbial, immunological and genetic factors that attribute to the progression of the disease. Numerous studies have been conducted in multiple aspects including clinical, endoscopy and histopathology for the diagnostics and treatment of IBD. However, the molecular mechanism underlying the aetiology and pathogenesis of IBD is still poorly understood. This review tries to critically assess the scientific evidence at the transcriptomic level as it would help in the discovery of RNA molecules in tissues or serum between the healthy and diseased or different IBD subtypes. These molecular signatures could potentially serve as a reliable diagnostic or prognostic biomarker. Researchers have also embarked on the study of transcriptome to be utilized in targeted therapy. We focus on the evaluation and discussion related to the publications reporting the different approaches and techniques used in investigating the transcriptomic changes in IBD with the intention to offer new perspectives to the landscape of the disease. (Intest Res 2018;16:374-383)

Key Words: Inflammatory bowel diseases; Colitis, ulcerative; Crohn disease; Transcriptome; Microarray analysis

\section{INTRODUCTION}

Inflammatory bowel disease (IBD) is a chronic, relapsing and remitting inflammatory gastrointestinal disorder comprises of 2 dominant sub-types which are CD and UC. CD affects any component of the gastrointestinal tract starting from the oral cavity to the anus with transmural inflammation. ${ }^{1,2}$ In contrast, UC affects the colon and characterized by the inflammation of the mucosa and submucosa layers of the rectum and colon accompanied by cyptitis. ${ }^{3}$ It is thought that several factors contributed to the development of the disease including environment, genetics, gut microbiota

Received December 6, 2017. Revised January 23, 2018. Accepted January 29, 2018. Published online April 6, 2018

Correspondence to Norfilza Mohd Mokhtar, Department of Physiology, Faculty of Medicine, Universiti Kebangsaan Malaysia Medical Centre, Jalan Yaacob Latif, Bandar Tun Razak, Cheras, Kuala Lumpur 56000, Malaysia. Tel: +60-3-9145-8610, Fax: +60-3-9145 8606, E-mail: norfilza@ppukm. ukm.edu.my and immune response to intestinal microbiota. ${ }^{2,45}$ However, at this point of time, there is an insufficient research data to make a definitive conclusion on the causes of IBD.

The incidence of CD has increased steadily in the North America and Europe ranged from 0 to 20.2 cases per 100,000 population while the incidence of UC ranged from 0 to 24.3 per 100,000 population. ${ }^{6}$ However, in recent years, there has been an increasing amount of literatures reporting on the sharp rise in the incidence of IBD in developing countries especially in Asia. ${ }^{7-10}$ The incidence of IBD in Asian countries ranged from 0.5 to 3 per 100,000 population with UC having a higher incidence as compared to $\mathrm{CD}$, approximately 1 and 0.5 per 100,000 populations respectively. ${ }^{11}$ Recent researches have suggested that urbanizations including Western dietary pattern, hygiene and childhood immunological factors are associated with IBD in Asia. ${ }^{12,13}$ Some other reports have identified that the changes in the gut microbiota and environmental factors are also linked to the causes of IBD., ${ }^{9,14,15}$

\footnotetext{
๑ Copyright 2018. Korean Association for the Study of Intestinal Diseases. All rights reserved.

This is an Open Access article distributed under the terms of the Creative Commons Attribution Non-Commercial License (http://creativecommons.org/licenses/by-nc/4.0)

which permits unrestricted non-commercial use, distribution, and reproduction in any medium, provided the original work is properly cited.
} 
However, apart from the aforementioned factors, a substantial number of studies had also reported on the involvement of genetic variations in the pathogenesis of IBD. ${ }^{16,17}$ There has been a surge of interest for the past few years in studying transcriptomic dysregulations in IBD whereby the changes involving all the transcribed RNA transcripts from the genomes. ${ }^{3,18,19}$ Transcriptomic studies are mainly dominated by arrays and sequencing technologies where vast information on the functions, expression levels and biological pathways of different transcripts are being analyzed with regards to IBD. ${ }^{18-21}$

\section{WHAT IS TRANSCRIPTOME?}

The term transcriptome is defined as a small proportion of the genetic codes that is transcribed into RNA molecules. ${ }^{22}$ It is estimated that less than $5 \%$ of the genome in human is transcribed into the RNA molecules. ${ }^{23}$ Interestingly, the proportion of transcribed RNA sequences that belongs to the nonprotein coding RNA groups appears to be in a greater percentage in a complex organism. ${ }^{24}$ Transcriptome is generated through the transcription process whereby DNA sequences are copied into complementary RNA strands by RNA polymerase. These complementary RNA strands, which consists of introns and exons, will undergo splicing to remove the introns in order to generate mature RNA transcripts that contain only the exons. ${ }^{25}$

Post-transcriptional RNA processing includes alternative splicing, RNA editing and various combinations of transcription initiation and termination sites are crucial processes in the cells as this could produce more than one variants of mRNA. In consequence, different protein products can be generated from a single transcribed gene. ${ }^{25}$ Therefore, investigations on the whole transcriptome would provide a better understanding on the complexity of the disease as compared to a single genomic study.

\section{DIFFERENT TYPES OF RNAS AND THEIR ASSOCIATIONS WITH DISEASES}

RNAs have been widely explored and have provided significant amount of information for a better understanding of IBD pathogenesis. RNAs are divided into different classes, including mRNA, transfer RNA (tRNA), ribosomal RNA (rRNA) and noncoding RNA (ncRNA). Examples include riboswitches, ribozymes, long noncoding RNA (IncRNA) and microRNA (miRNA). ${ }^{26}$ A diagrammatic representations of different classes of RNAs in eukaryotic cells involved in
IBD is as shown in Fig. 1. Mature mRNA contains coding information for only one polypeptide chain which comprises of a cap, a coding region with exons and a tailing sequence that includes the poly(A) tail. ${ }^{27}$ The translation of mature mRNA into its specific protein is controlled by poly (A)binding protein that binds to the mRNA's poly A tail. ${ }^{28}$ Based on these RNA characteristics, advanced techniques such as next-generation sequencing (NGS), transcriptomic array, TaqMan gene expression array and microarray were established and used in the high throughput data analysis of mRNA. ${ }^{3,16,29,30}$ As mRNAs contain information for proteins formation and different pathways regulation, an increasing amount of researches have been accomplished worldwide in exploring the transcripts sequences, functions, translation, expression level and their influences in different kind of diseases. ${ }^{31-33}$ Examples of diseases where their pathogenesis had been studied in terms of the dysregulations of mRNAs include schizophrenia, cancer, autoimmunity, neurological disorders and diabetes. ${ }^{17,34,35}$ In transcriptomic studies of IBD, most of the aberrant mRNAs were found to be involved in molecular functions associated with immune response, mucosal inflammation, nutrients absorption, epithelial damage, oncogenesis and cell proliferation. .1,36,37 $^{-1}$

rRNA, which is a part of ribosome (a protein synthesizing organelle), plays an important role in translating the information in mRNA to protein in the cytoplasm in a process called translation. In human genome, there are approxi-

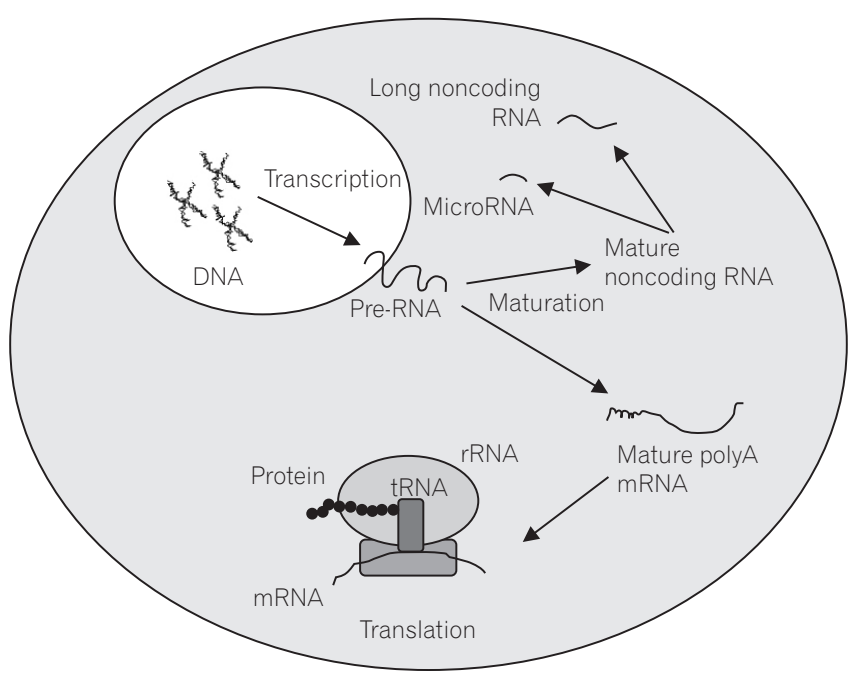

Fig. 1. A representation of different types of RNAs in eukaryotic cells. Different classes of matured RNAs transcribed from genomic DNA are involved in the regulation of different cellular processes and their dysregulations could lead to numerous diseases including IBD. rRNA ribosomal RNA; tRNA, transfer RNA. 
mately 300 to 400 copies of rRNA genes and they are organized in a repeated tandem arrays within nucleolar organizing regions located on the short arm of chromosome 13, 14, 15,21 and $22 .{ }^{38}$ Variations in rRNA genes and its expression level has been reported to be associated with carcinogenesis, pathogenesis of schizophrenia and autism as well as in hearing loss. ${ }^{39,40}$

tRNAs are the adaptor molecules for accurate mRNA translation for protein synthesis. The mature form of tRNAs consists of approximately 70 to 100 nucleotides long known as "clover" leaf secondary structure and folds into a common L-shaped architecture. ${ }^{41}$ These amino acid carriers decode the nucleotide sequence of mRNA into specific polypeptide sequences thus allowing the genetic code to be specifically translated for protein synthesis. ${ }^{42}$ Additional role of tRNAs have been reported in stress response, gene regulation and plasmid replication. ${ }^{43,44}$ Similarly to rRNA study, there has been no report on the involvement of the tRNA on the pathogenesis of IBD.

ncRNAs are described as RNA molecules that are nontemplates for protein synthesis and are expressed as small or IncRNAs. ${ }^{45}$ IncRNAs are generally defined as ncRNAs that are greater than 200 nucleotides in length. ${ }^{46}$ A great deal of previous researches into IncRNAs have focused on the functions

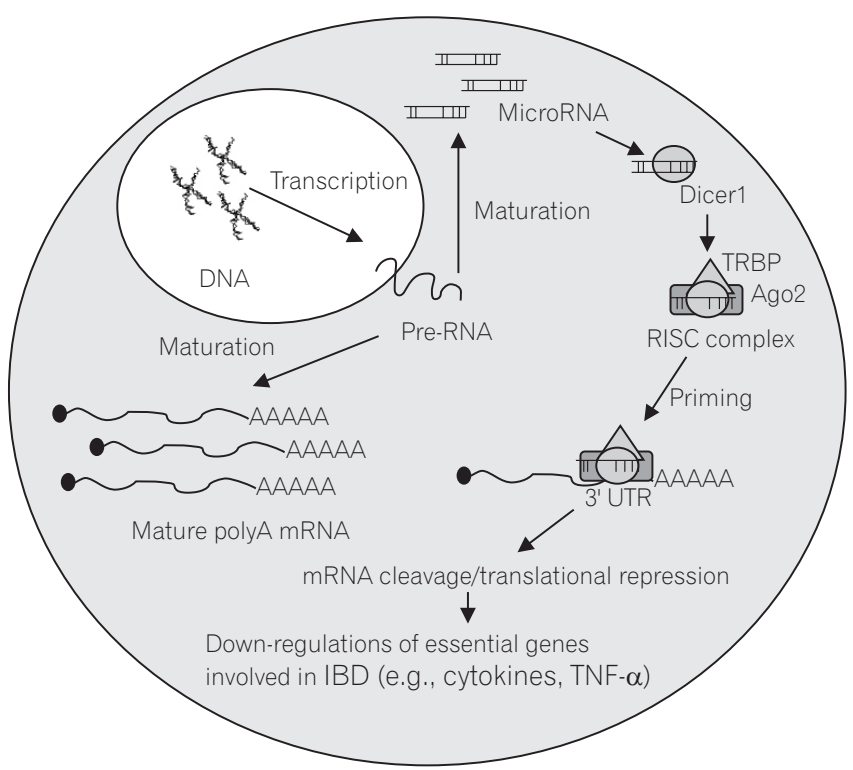

Fig. 2. Mechanism of action of microRNA in repressing the essential genes involved in the regulation of inflammatory processes in epithelial cells through the binding at the 3' UTR of the genes. Dicer1, RNAse endonuclease; RISC complex, RNA-induced silencing complex; TRBP, protein with 3 double-standard RNA-binding domains; Ago2, Argonaute 2; 3' UTR, 3' untranslated regions; TNF- $\alpha$, tumour necrosis factor $\alpha$. of this molecule on numerous cellular processes including cell cycle regulation, stem cell pluripotency, retrotransposon silencing, meiotic entry and telomere length. ${ }^{47,48}$ Thus, it is reported that lncRNAs are able to regulate gene expressions including the neighboring protein-coding genes. ${ }^{49-51}$ In IBD, overexpression of IncRNAs were reported to be related to immune response, pro-inflammatory cytokines activity and major histocompatibility protein complex. ${ }^{3}$

miRNAs belong to a family of small ncRNAs (20-24 bps) and play important roles in human as they are involved in the post-transcriptional stage of gene expression. ${ }^{52,53}$ miRNA represses gene function by binding at the 3 ' untranslated region (UTR) of the targeted mRNA and subsequently leads to the prevention of mRNA translation or degradation (Fig. 2 ). The roles of miRNAs have been described in cellular processes including proliferation, apoptosis, development and cell fate programming. ${ }^{54-56}$ It has been demonstrated that altered miRNAs expressions are observed in a number of diseases such as in cancer and cardiovascular disorders, myocardial infarction, heart failure, acute coronary syndrome and IBD. ${ }^{57-59}$ Numerous miRNAs were found to be differentially expressed in IBD and were hypothesized to act as critical mediators in the IBD pathogenesis by regulating proteins in vital regulatory pathways such as in the expression of inflammatory cytokines. ${ }^{60}$

\section{TRANSCRIPTOMIC DYSREGULATION STUDIES IN IBD}

Various experimental designs on the study of transcriptomic dysregulations have been tailored and carried out in IBD patients whereby novel findings, objectives or correlations between the studied cohorts could be discovered in terms of the changes in RNAs expression. All the different approaches and studies discussed here are summarized in Table 1.,18,21,37,61,62 One of the common achievements includes the genome-wide microarray study identified different types of aberrant RNAs and the deregulation of molecular mechanisms directly involved in IBD, including the complex array of pathways from inflammatory responses to the deregulations of growth factors. For example, a comprehensive high-throughput mRNA expression study by Costello et al. ${ }^{37}$ had reported that a total of 500 and 272 transcripts were differentially regulated in CD and UC patients respectively. The gene dysregulation study was conducted using genome-wide cDNA microarrays with biopsies taken from normal ( $\mathrm{n}=11), \mathrm{CD}(\mathrm{n}=10)$ and UC $(\mathrm{n}=10)$ patients followed by verification of the gene expression of interesting hits with 


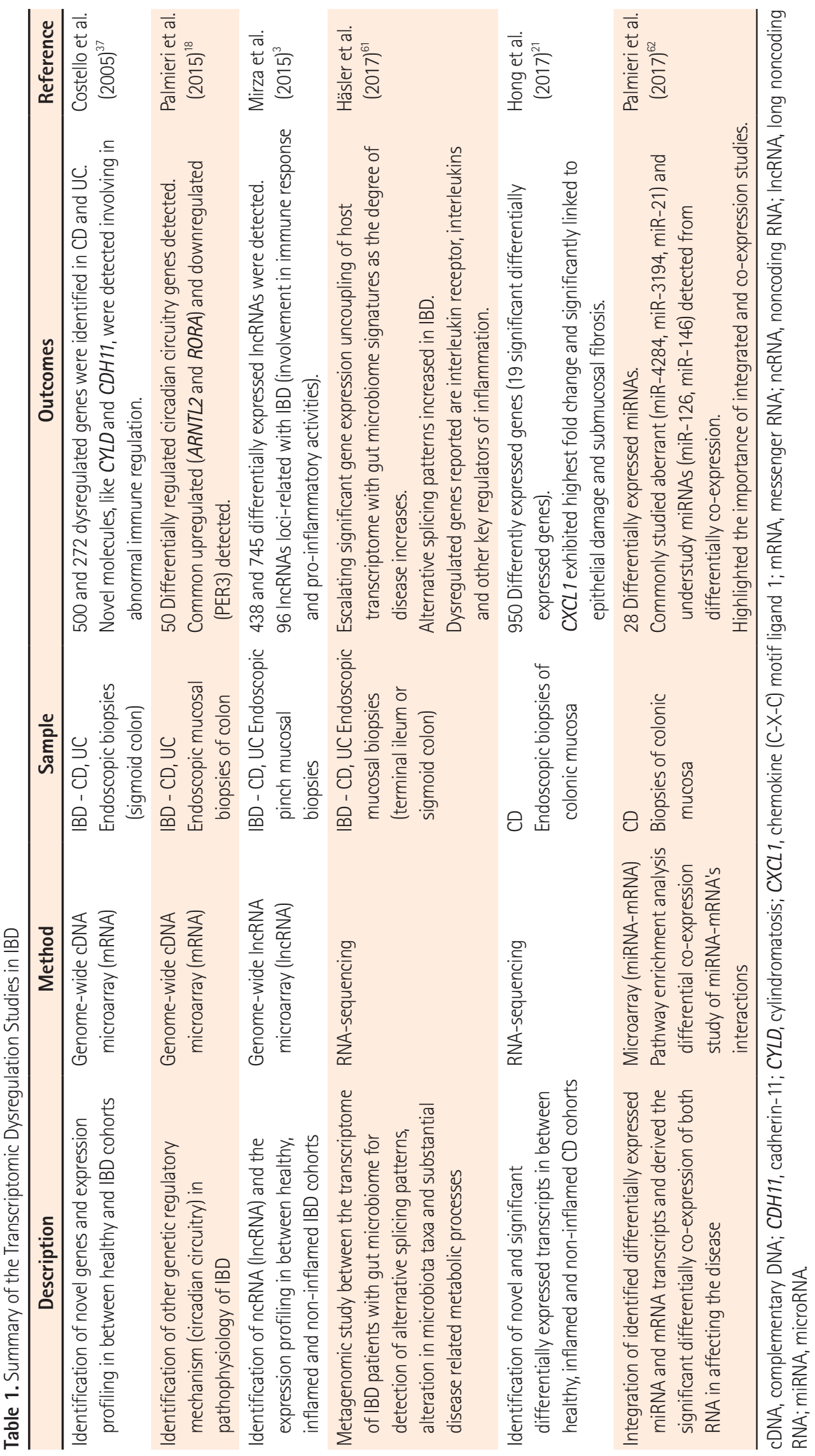


real-time quantitative PCR (qPCR) in additional samples of 100 individuals. From the results, there were 122 genes found to be dysregulated in both conditions and on average $\sim 40 \%$ of these identified differentially regulated genes are novel and unannotated ${ }^{37}$ These differentially upregulated genes were found to be involved in the functional category of "immune and inflammatory response," "oncogenesis," "cell proliferation and growth" and "structure and permeability" whereby a transcribed gene sequence with strong similarity to protein pir:I38067, nitric-oxide synthase, and the Ig heavy constant $\gamma 1$ (IGHGI) gene is reported to be upregulated by more than 10-fold in both IBD cases against normal patients. While for another aberrant gene, Ig heavy locus (IGH@), it was upregulated by more than 10 -fold in UC and 5.5 -fold in $\mathrm{CD}$ against the control. They also reported that cadherin-11 (CDH11), decay accelerating factor for complement $(D A F)$, mucin 1 (MUC1), phospholipase A2, group IIA (PLA2G2A), and tissue inhibitor of metalloproteinase 1 (TIMP1) were upregulated in both IBD subtypes. ${ }^{37}$ In this study, they also reported the discovery of an unknown gene, DKFZp547A023, which was subsequently confirmed by qPCR to be downregulated in both disease groups and it could serve as a potential candidate for further studies. As for the downregulated genes, their microarray results showed that cylindromatosis $(C Y L D)$, calcitonin gene-related peptide receptor component protein (RCP9), LIM protein (LIM), occluding (OCLN), Rho-associated, coiled-coil containing protein kinase 1 (ROCK1), and zinc finger, CCHC domain containing 4 ( $Z C$ CHC4) were involved in both IBD subtypes. ${ }^{37}$

Besides that, whole transcriptomic study on the identifications of aberrant RNAs also provides insightful correlations of other regulatory mechanisms which might be involved in the aetiopathogenesis of IBD. In a genome-wide cDNA microarray study by Palmieri et al. ${ }^{18}$ on the mucosal biopsies samples of 29 IBD patients ( 15 CD and 14 UC) has reported that out of 150 circadian genes, 21 genes (14\%) in CD and 27 genes (18\%) in UC showed upregulation, whereas 29 genes (19\%) in CD and 23 (15\%) in UC showed downregulation. Among the reported genes, $A R N T L 2$, the core clock gene and RORA, nuclear hormones receptor were reported to be upregulated while PER3, the clock gene was downregulated in both conditions. As circadian clock circuitry is involved in the regulation of important cell processes and organ functions, changes in circadian cycle is involved in the basic mechanism of inflammatory and neoplastic diseases. ${ }^{18}$ It has also been suggested that dysregulated circadian cycle altered sleep patterns in UC and CD patients whereby this could affect the severity of the disease through the immune system activation and the release of inflammatory cytokines. $^{63}$

Researchers were also trying to explore into the roles of IncRNAs in IBD, apart from focusing only on the mRNAs studies. For example, Mirza et al. ${ }^{3}$ had conducted a genomewide study on IncRNAs and protein-coding genes profiling by microarray platform on inflamed and non-inflamed pinch biopsies from IBD patients (13 CD and 20 UC) and controls $(\mathrm{n}=12)$. They reported that there were 254 upregulated and 184 downregulated IncRNAs in inflamed CD and 370 upregulated and 375 downregulated lncRNAs in UC as compared to the control. Besides, there were 31 and 19 differentially expressed lncRNAs identified in the non-inflamed CD and UC against the control. The lncRNAs included RP11-731 F5.2, MMP12 and RP11-465 L10.10 were identified among the highly upregulated IncRNAs in both inflamed CD and UC against the control. ${ }^{3}$ DPP10-AS1, PDZK1P2 and antisense non-coding RNA in the INK4 locus (ANRIL) were reported to be downregulated while dual oxidase 2 (DUOX2) and its maturation factor, dual oxidase maturation factor 2 (DUOXA2), has been reported to be upregulated in association with inflamed UC. ${ }^{3}$ Their results concluded that the functional groupings of the most differentially expressed IncRNAs were enriched in the area of immune response and pro-inflammatory cytokine activity, suggested the involvement of this RNA in the persistent inflammation and pathogenesis of IBD.

A study on inflamed, non-inflamed and healthy mucosa layers of CD patients, Hong et al. ${ }^{21}$ had investigated the transcriptomic differences in terms of mRNA in between the cohorts in $13 \mathrm{CD}$ patients (male, $\mathrm{n}=7$ ) against 13 gendermatch healthy individuals using RNA-sequencing (RNASeq) instead of microarray. About 950 genes were reported to be differentially regulated, 19 genes were detected to be significantly differentially expressed with chemokine (C-XC) motif ligand 1 (CXCL1) gene reported to show the highest upregulation among them. Immunohistochemistry assay and qPCR further confirmed that CXCL1 gene expression was significantly steadily increased from no expression in normal mucosa to increased expression in inflamed CD mucosa. CXCL1 is a type of CXC chemokine which is known to be responsible in the accumulation of polymorphonuclear leukocytes for acute inflammation and infiltration of the inflamed tissue. ${ }^{21}$ Apart from being able to provide new findings on the roles of different RNAs in disease progression, the transcriptome studies on different RNAs (such as in mRNAs and InRNAs) on a similar research backgrounds are important. This is because although the experiments were 
conducted by different groups, these results could be integrated and offer new perspectives on how the different RNAs interact and affecting the meta-omic environment of IBD.

In realizing the importance of understanding the complementation roles played by the different types of RNAs in meta-omic IBD research, recent experimental approach in etiopathology transcriptomic studies can be seen to adopt a more integrative model where the relationships of the paired expressions changes of different RNAs were investigated. ${ }^{62}$ A study by Palmieri et al. documented the integration of microarray expression profiles of ncRNAs and mRNAs in inflamed tissues of $15 \mathrm{CD}$ patients relative to the normal mucosa. Additionally, they reported a more relevant and meaningful functional implications based on the paired coexpressions to the extent of pathway related to the disease through a computational statistical procedure. For example, they reported that miR-21, miR-126, miR-146a and miR3194 possessed functional roles in CD as they were found to be differentially expressed and differentially co-expressed in the platelet activation signaling and aggregation pathway, one of the crucial dysregulated pathways in IBD. Through their analyses, they also discovered that some miRNAs that were differentially expressed did not involve in the differential co-expression with mRNA in the dysregulated biological processes pathways in IBD. On the other hand, some of the miRNAs that were not among the significantly differentially expressed list were found to be involved in the dysregulated pathways in IBD.

Other integrative experimental approaches such as metagenomic had also been reported in IBD studies. Häsler et al. ${ }^{61}$ investigated the relationship between the transcriptome changes in IBD patients with the microbiome of gut adherent mucosa-microbiota. The transcriptomic changes with regards to intestinal inflammation comparing between healthy and non-IBD inflamed control groups using NGS were determined in 63 biopsies ( 12 healthy, 15 disease control, 17 UC and 19 CD) in 41 individuals. Disease control groups were used to differentiate phenomenon to be IBDspecific and it is defined as infectious acute non-IBD inflammation. Intestinal microbiota has been known to play a role in shaping the immune response and other physiological functions in the intestinal environment. Any defective interactions between them could lead to the progression of IBD. Hence, Häsler et al. ${ }^{61}$ studied the host's transcriptomic responses and changes in the context of the microbiota signatures, inflammation and alternative splicing events in the development of the disease as they hypothesized that these could provide more implications on the understanding of the etiopathology of IBD rather than focusing on the level of differentially expressed genes alone. They discovered that there was no connection between gene expression of the host and the microbiota transcriptome in the inflamed colonic mucosa of IBD patients as compared to the non-IBD inflammation and healthy cohorts. Differential expressed genes were found to be involved in the pathophysiology of IBD such as interleukin-1 receptor type II, interleukin-6, and interleukin-8 while genes which showed alternative splicing were $D U O X 2$, autophagy-related 16-like 1 and various interleukins. The most relevant pathway was microbiota-related inflammation pathway such as the chemokine signaling, natural killer cell-mediated cytotoxicity, NOD-like receptor signaling and spliceosome assembly. ${ }^{61}$

\section{ADVANTAGES AND LIMITATIONS OF TRANSCRIPTOMIC STUDIES IN IBD}

The reports of transcriptomic research in IBD are increasing as it provides evidence on the role of protein-coding mRNA and ncRNAs in modulating immune response in $\mathrm{IBD}^{64,65}$ In transcriptomic analysis, microarray and RNASeq techniques have been reported to be the current stateof-the-art and they had been widely utilized in studies with the effort to understand the etiology of IBD, especially on immune regulatory and signaling mechanisms. ${ }^{3,66}$ Furthermore, the high correlations between the high-throughput genome-wide association study (GWAS) data with independent transcriptomic data in IBD sample studies were reported to reveal the importance of gene expression signatures especially in inflammatory-related pathway. ${ }^{67}$ This had identified deregulated mRNAs and ncRNAs that are potentially utilized as biomarkers for the diagnostic of disease or served as targets in the discovery of novel therapeutic strategies. Nonetheless, there is also an example of study that showed the regulation of the pathways that are not detected by GWAS. A study by Cardinale et al. ${ }^{67}$ demonstrated that the downregulation in oxidative phosphorylation and upregulation of mitotic control pathways were detected in the gene expression analysis but not in GWAS study. Thus, although a huge amount of data could be obtained through GWAS study, independent transcriptomic studies are important to complement and validate the results obtained in GWAS related to IBD research. Moreover, the results from the transcriptomic study could only provide information on the putative identity of the aberrant RNAs. 


\section{MICROARRAY ANALYSIS VERSUS RNA- SEQUENCING IN TRANSCRIPTOMIC STUDY}

Microarray and RNA sequencing are employed in most of the transcriptomic studies, especially in researches to investigate the molecular pathogenesis mechanism in human diseases. By looking at the summary of the discussed studies (Table 1), the earlier IBD transcriptomic studies were dominated by microarray analysis and utilization of the RNA sequencing technique only gained popularity in the recent years. Microarray analysis is a high-throughput simultaneous screening method of multiple targets arranged in a microchip while RNA sequencing is a process involving multiple stages including amplifications, sequencing, assembly and mapping from the whole transcriptome. While both techniques are commonly used to identify the transcript populations and their expression profiles in the sample, each of the techniques has their own strengths and weaknesses.

For the transcript identifications in microarray analysis, the arranged probes in the microchip are designed based on the currently available genomic sequences, whereas in RNA sequencing, each of the transcripts in the wh0ole transcriptome are sequenced and subsequently identified through reads assemblies and mapping. ${ }^{21,66}$ Transcripts identification in the microarray analysis is restricted to the detection of transcripts with known genomic sequences while transcripts of both known and unknown (novel) sequences can be identified in the same sample source using RNA sequencing. ${ }^{21}$ Besides, it has been suggested that the results from RNA sequencing possesses higher reproducibility, better measurements of gene expressions level and additional information on isoforms can be provided as compared to the array method. ${ }^{21,66,68}$ In RNA sequencing, specific read sequences of the splice junctions can be obtained and this leads to the direct identifications of known and also novel alternative splicing forms.

Performing RNA sequencing analysis is much more complicated than the microarray as there are more steps involved in the whole process. It would require a bioinformaticians or personnel experienced in bioinformatics to process and analyze the massive generated raw reads. The bioinformatics software often needs optimizations especially on the processes involved in analyzing the raw data. This includes processes like efficient data storage and retrieval, minimizing errors in image analysis, base calling and removal of lowquality reads before continuing with mapping and transcript assembly. While there are numerous tools developed and available to proceed with the analysis, the most suitable pro- gram has to be selected carefully depending on the project as different research questions cannot be answered and analyzed using similar bioinformatics tools. ${ }^{66}$

Microarray has been the commonly used technique to study transcriptomes in healthy condition or diseases before the advent of RNA sequencing. It is suitable to be used in expression profiling investigations with a defined pool of genes of interest, in performing targeted gene expression studies and in searching for selected alternatively spliced sequences. The volume of the data generated from the microarray analysis is high-throughput with relatively cheaper in term of cost per sample as compared to the RNA sequencing. ${ }^{66}$ In order to obtain a comprehensive representation of gene expression or for the detection of rare transcripts from complex organisms, repeated runs or more sequencing depth would be required in the sequencing of the samples. The need for repeated sequencing or more sequencing depth would imply a higher sequencing cost in RNA sequencing as compared to microarray analysis. In general, performing a RNA sequencing study would be more expensive and complicated than the microarray analysis and the most cost effective method should be decided based on the objectives of the experiment.

The problems that people face in microarray analysis relate with probe hybridization behavior, cross hybridization of similar sequence probes and in differentiating the signal-tonoise ratios even though custom arrays with specific probes of known sequences are used ${ }^{68}$ The dependence of microarray analysis on known genomic sequences and the presence of high background noise due to cross-hybridizations had limited the detection depth of gene isoforms and alternative spliced transcripts species. ${ }^{66}$ Following this, the quantifications, detection sensitivity and calculated abundance of the transcripts could be compromised due to artefacts formations and subsequently affect the accuracy of genes expression level deteminations. ${ }^{68}$

\section{FUTURE RESEARCH DIRECTIONS}

Based on the current reported studies, transcriptome expression profiling studies had discovered numerous aberrant RNAs which are potentially involved in the pathogenesis of IBD. This information could be utilized as targets in the development of diagnostic biomarkers or therapeutic agents in IBD treatments. Microarray and NGS techniques are proven to be a useful tool in high-throughput genomewide gene expression studies with verification studies using qPCR. More experimental designs incorporating transcrip- 
tomic studies could be conducted to further reveal on the molecular mechanisms and characterizations of RNAs in IBD by different study cohorts such as on the effects of disease duration of IBD patients leading to neoplasms. Knowledge on the changes in transcriptome could answer on how the different novel treatments could affect the progression of the disease. Functional validation studies are ought to be conducted to further confirm the roles played by these identified aberrant RNAs before incorporating them to any therapeutic treatments. While there have been several studies validating the functions of the aberrant RNAs in vitro, ${ }^{69,70}$ there are relatively less functional studies being conducted in vivo. ${ }^{71}$ This is important as without knowing the functions of the discovered RNAs, targeted therapeutic approaches cannot be designed effectively. Although in vitro methods are commonly used in the functional validations, it is better to be complement with the more reliable in vivo validations using an animal models of IBD. ${ }^{72}$

\section{CONCLUSIONS}

High-throughput transcriptomic profiling techniques has become widely available serving as the common platform for data analysis and opportunities for the scientists to obtain a comprehensive understanding of IBD in term of genetic makeup. Transcriptomic analysis approach gained popularity due to their potentials towards identification of changes in the different RNA transcripts including novel and unknown transcripts underlying the IBD. More evidencebased researches will help to achieve a better understanding on the pathogenesis, etiology and complications of the complex and challenging disease such as IBD.

\section{FINANCIAL SUPPORT}

This research was supported from Fundamental Research Grant Scheme (FRGS)-FRGS/1/2015/SKK08/UKM/02/2 and Geran Universiti Penyelidikan (GUP)-GUP-2017-090.

\section{CONFLICT OF INTEREST}

No potential conflict of interest relevant to this article was reported.

\section{AUTHOR CONTRIBUTION}

Conceptualizations: SNC, NMM. Writing-original draft: SNC, NMM. Writing-review and editing: SNC, NMM, RARA.
Approval of final manuscripts: all authors.

\section{REFERENCES}

1. Abraham C, Cho JH. Inflammatory bowel disease. N Engl J Med 2009;361:2066-2078.

2. Fiocchi C. Inflammatory bowel disease: etiology and pathogenesis. Gastroenterology 1998;115:182-205.

3. Mirza AH, Berthelsen CH, Seemann SE, et al. Transcriptomic landscape of lncRNAs in inflammatory bowel disease. Genome Med 2015;7:39. doi: 10.1186/s13073-015-0162-2.

4. Kaser A, Zeissig S, Blumberg RS. Inflammatory bowel disease. Annu Rev Immunol 2010;28:573-621.

5. Zhang YZ, Li YY. Inflammatory bowel disease: pathogenesis. World J Gastroenterol 2014;20:91-99.

6. Ananthakrishnan AN. Epidemiology and risk factors for IBD. Nat Rev Gastroenterol Hepatol 2015;12:205-217.

7. Hu PJ. Inflammatory bowel disease in Asia: the challenges and opportunities. Intest Res 2015;13:188-190.

8. Prideaux L, Kamm MA, De Cruz PP, Chan FK, Ng SC. Inflammatory bowel disease in Asia: a systematic review. J Gastroenterol Hepatol 2012;27:1266-1280

9. Ng SC. Epidemiology of inflammatory bowel disease: focus on Asia. Best Pract Res Clin Gastroenterol 2014;28:363-372.

10. Ali RA. The positive influences of increasing age at diagnosis of inflammatory bowel disease on disease prognostication in Asian perspective. Intest Res 2015;13:4-5.

11. Ng SC. Emerging trends of inflammatory bowel disease in Asia. Gastroenterol Hepatol (N Y) 2016;12:193-196.

12. Ng SC, Tang W, Ching JY, et al. Incidence and phenotype of inflammatory bowel disease based on results from the Asiapacific Crohn's and colitis epidemiology study. Gastroenterology 2013;145:158-165.e2.

13. Kanai T, Matsuoka K, Naganuma M, Hayashi A, Hisamatsu T. Diet, microbiota, and inflammatory bowel disease: lessons from Japanese foods. Korean J Intern Med 2014;29:409-415.

14. Ng SC. Emerging leadership lecture: inflammatory bowel disease in Asia: emergence of a "Western" disease. J Gastroenterol Hepatol 2015;30:440-445.

15. Cheon JH. Genetics of inflammatory bowel diseases: a comparison between Western and Eastern perspectives. J Gastroenterol Hepatol 2013;28:220-226.

16. Söderman J, Berglind L, Almer S. Gene Expression-genotype analysis implicates GSDMA, GSDMB, and LRRC3C as contributors to inflammatory bowel disease susceptibility. Biomed Res Int 2015;2015:834805. doi: 10.1155/2015/834805. 
17. Xu XR, Liu CQ, Feng BS, Liu ZJ. Dysregulation of mucosal immune response in pathogenesis of inflammatory bowel disease. World J Gastroenterol 2014;20:3255-3264.

18. Palmieri O, Mazzoccoli G, Bossa F, et al. Systematic analysis of circadian genes using genome-wide cDNA microarrays in the inflammatory bowel disease transcriptome. Chronobiol Int 2015;32:903-916.

19. Fang K, Grisham MB, Kevil CG. Application of comparative transcriptional genomics to identify molecular targets for pediatric IBD. Front Immunol 2015;6:165. doi: 10.3389/fimmu.2015.00165.

20. Planell N, Lozano JJ, Mora-Buch R, et al. Transcriptional analysis of the intestinal mucosa of patients with ulcerative colitis in remission reveals lasting epithelial cell alterations. Gut 2013;62:967-976.

21. Hong SN, Joung JG, Bae JS, et al. RNA-seq reveals transcriptomic differences in inflamed and noninflamed intestinal mucosa of Crohn's disease patients compared with normal mucosa of healthy controls. Inflamm Bowel Dis 2017;23:1098-1108.

22. Adams J. Transcriptome: connecting the genome to gene function. Nat Educ 2008;1:195.

23. Frith MC, Pheasant M, Mattick JS. The amazing complexity of the human transcriptome. Eur J Hum Genet 2005;13:894-897.

24. Mathew S, Shaabad M, Hussein S, Mira L, Qadri I. The need behind messenger RNA sequencing analysis. Int J Adv Res 2015;3:1260-1270.

25. Elliott DJ. Illuminating the transcriptome through the genome. Genes (Basel) 2014;5:235-253.

26. Wan Y, Kertesz M, Spitale RC, Segal E, Chang HY. Understanding the transcriptome through RNA structure. Nat Rev Genet 2011;12:641-655.

27. Peet A, Lieberman M, Marks AD. Marks' basic medical biochemistry (Lieberman, Marks's basic medical biochemistry). 4th ed. Philadelphia: Lippincott Williams \& Wilkins; 2013.

28. Gray NK, Hrabálková L, Scanlon JP, Smith RW. Poly(A)-binding proteins and mRNA localization: who rules the roost? Biochem Soc Trans 2015;43:1277-1284.

29. Holgersen K, Kutlu B, Fox B, et al. High-resolution gene expression profiling using RNA sequencing in patients with inflammatory bowel disease and in mouse models of colitis. J Crohns Colitis 2015;9:492-506.

30. Zhang T, Song B, Zhu W, et al. An ileal Crohn's disease gene signature based on whole human genome expression profiles of disease unaffected ileal mucosal biopsies. PLoS One 2012;7:e37139. doi: 10.1371/journal.pone.0037139.

31. Wu F, Dong F, Arendovich N, Zhang J, Huang Y, Kwon JH. Divergent influence of microRNA-21 deletion on murine colitis phenotypes. Inflamm Bowel Dis 2014;20:1972-1985.
32. Li E, Hamm CM, Gulati AS, et al. Inflammatory bowel diseases phenotype, C. difficile and NOD2 genotype are associated with shifts in human ileum associated microbial composition. PLoS One 2012;7:e26284. doi: 10.1371/journal.pone.0026284.

33. van Lierop PP, Swagemakers SM, de Bie CI, et al. Gene expression analysis of peripheral cells for subclassification of pediatric inflammatory bowel disease in remission. PLoS One 2013;8:e79549. doi: 10.1371/journal.pone.0079549.

34. Zhao Z, Jinde S, Kakiuchi C, Kasai K. Extracellular elevation of adrenomedullin, a gene associated with schizophrenia, suppresses heat shock protein 1A/1B mRNA. Neuroreport 2016;27:1312-1316.

35. Lee TI, Young RA. Transcriptional regulation and its misregulation in disease. Cell 2013;152:1237-1251.

36. Xu L, Ma L, Lian J, Yang J, Chen S. Gene expression alterations in inflamed and unaffected colon mucosa from patients with mild inflammatory bowel disease. Mol Med Rep 2016;13:27292735 .

37. Costello CM, Mah N, Häsler R, et al. Dissection of the inflammatory bowel disease transcriptome using genome-wide cDNA microarrays. PLoS Med 2005;2:e199. doi: 10.1371/journal. pmed.0020199.

38. Karahan G, Sayar N, Gozum G, Bozkurt B, Konu O, Yulug IG. Relative expression of rRNA transcripts and 45S rDNA promoter methylation status are dysregulated in tumors in comparison with matched-normal tissues in breast cancer. Oncol Rep 2015;33:3131-3145.

39. Porokhovnik LN, Pasekov VP, Egolina NA, et al. Oxidative stress, rRNA genes, and antioxidant enzymes in pathogenesis of schizophrenia and autism: modeling and clinical advices. Zh Obshch Biol 2013;74:340-353.

40. Peng GH, Fang F, Zheng J, et al. Mitochondrial 12S rRNA variants studies in 456 subjects with hearing loss in seven schools for deaf and mutes in Zhejiang province. Zhonghua Er Bi Yan Hou Tou Jing Wai Ke Za Zhi 2012;47:996-1003.

41. Torres AG, Batlle E, Ribas de Pouplana L. Role of tRNA modifications in human diseases. Trends Mol Med 2014;20:306-314.

42. Giegé R, Jühling F, Pütz J, Stadler P, Sauter C, Florentz C. Structure of transfer RNAs: similarity and variability. Wiley Interdiscip Rev RNA 2012;3:37-61.

43. Thompson DM, Lu C, Green PJ, Parker R. tRNA cleavage is a conserved response to oxidative stress in eukaryotes. RNA 2008;14:2095-2103.

44. Melançon CE 3rd, Schultz PG. One plasmid selection system for the rapid evolution of aminoacyl-tRNA synthetases. Bioorg Med Chem Lett 2009;19:3845-3847. 
45. Novikova IV, Hennelly SP, Tung CS, Sanbonmatsu KY. Rise of the RNA machines: exploring the structure of long non-coding RNAs. J Mol Biol 2013;425:3731-3746.

46. Bassett AR, Akhtar A, Barlow DP, et al. Considerations when investigating lncRNA function in vivo. Elife 2014;3:e03058. doi: 10.7554/eLife.03058.

47. Shoemaker DD, Schadt EE, Armour CD, et al. Experimental annotation of the human genome using microarray technology. Nature 2001;409:922-927.

48. Lander ES, Linton LM, Birren B, et al. Initial sequencing and analysis of the human genome. Nature 2001;409:860-921.

49. Mercer TR, Neph S, Dinger ME, et al. The human mitochondrial transcriptome. Cell 2011;146:645-658.

50. Kornienko AE, Guenzl PM, Barlow DP, Pauler FM. Gene regulation by the act of long non-coding RNA transcription. BMC Biol 2013;11:59. doi: 10.1186/1741-7007-11-59.

51. Muers M. RNA: genome-wide views of long non-coding RNAs. Nat Rev Genet 2011;12:742. doi: 10.1038/nrg3088.

52. Fabian MR, Sonenberg N, Filipowicz W. Regulation of mRNA translation and stability by microRNAs. Annu Rev Biochem 2010;79:351-379.

53. Schanen BC, Li X. Transcriptional regulation of mammalian miRNA genes. Genomics 2011;97:1-6.

54. Chen Y, Stallings RL. Differential patterns of microRNA expression in neuroblastoma are correlated with prognosis, differentiation, and apoptosis. Cancer Res 2007;67:976-983.

55. Lüningschrör P, Hauser S, Kaltschmidt B, Kaltschmidt C. MicroRNAs in pluripotency, reprogramming and cell fate induction. Biochim Biophys Acta 2013;1833:1894-1903.

56. Bartel DP. MicroRNAs: target recognition and regulatory functions Cell. 2009;136:215-233.

57. Lages E, Ipas H, Guttin A, Nesr H, Berger F, Issartel JP. MicroRNAs: molecular features and role in cancer. Front Biosci (Landmark Ed) 2012;17:2508-2540.

58. Di Stefano V, Zaccagnini G, Capogrossi MC, Martelli F. MicroRNAs as peripheral blood biomarkers of cardiovascular disease. Vascul Pharmacol 2011;55:111-118.

59. Tijsen AJ, Creemers EE, Moerland PD, et al. MiR423-5p as a circulating biomarker for heart failure. Circ Res 2010;106:10351039.

60. Kim HY, Kwon HY, Thi HT, et al. MicroRNA-132 and microRNA-223 control positive feedback circuit by regulating FOXO3a in inflammatory bowel disease. J Gastroenterol Hepatol 2016;31:1727-1735.
61. Häsler R, Sheibani-Tezerji R, Sinha A, et al. Uncoupling of mucosal gene regulation, mRNA splicing and adherent microbiota signatures in inflammatory bowel disease. Gut 2017;66:20872097.

62. Palmieri O, Creanza TM, Bossa F, et al. Functional implications of microRNAs in Crohn's disease revealed by integrating microRNA and messenger RNA expression profiling. Int J Mol Sci 2017;18:E1580. doi: 10.3390/ijms18071580.

63. Swanson GR, Burgess HJ, Keshavarzian A. Sleep disturbances and inflammatory bowel disease: a potential trigger for disease flare? Expert Rev Clin Immunol 201 1;7:29-36.

64. Pekow JR, Kwon JH. MicroRNAs in inflammatory bowel disease. Inflamm Bowel Dis 2012;18:187-193.

65. Coskun M, Bjerrum JT, Seidelin JB, Troelsen JT, Olsen J, Nielsen OH. miR-20b, miR-98, miR-125b-1*, and let-7e* as new potential diagnostic biomarkers in ulcerative colitis. World J Gastroenterol 2013;19:4289-4299.

66. Wang Z, Gerstein M, Snyder M. RNA-Seq: a revolutionary tool for transcriptomics. Nat Rev Genet 2009;10:57-63.

67. Cardinale CJ, Wei Z, Li J, et al. Transcriptome profiling of human ulcerative colitis mucosa reveals altered expression of pathways enriched in genetic susceptibility loci. PLoS One 2014;9:e96153. doi: 10.1371/journal.pone.0096153.

68. Richard H, Schulz MH, Sultan M, et al. Prediction of alternative isoforms from exon expression levels in RNA-Seq experiments. Nucleic Acids Res 2010;38:e112. doi: 10.1093/nar/gkq041.

69. Kanaan Z, Rai SN, Eichenberger MR, et al. Differential microRNA expression tracks neoplastic progression in inflammatory bowel disease-associated colorectal cancer. Hum Mutat 2012;33:551-560.

70. Olaru AV, Yamanaka S, Vazquez C, et al. MicroRNA-224 negatively regulates p21 expression during late neoplastic progression in inflammatory bowel disease. Inflamm Bowel Dis 2013;19:471-480.

71. Polytarchou C, Hommes DW, Palumbo T, et al. MicroRNA214 Is associated with progression of ulcerative colitis, and inhibition reduces development of colitis and colitis-associated cancer in mice. Gastroenterology 2015;149:981-992.e11.

72. Saeidnia S, Manayi A, Abdollahi M. From in vitro experiments to in vivo and clinical studies; pros and cons. Curr Drug Discov Technol 2015;12:218-224. 